\title{
Relief of pain in Parkinson disease
}

Parkinson disease (PD) is primarily recognized as a motor disorder: rest tremor, rigidity and bradykinesia are key clinical features used to characterize the disease. Many secondary symptoms accompany these major signs, however, including pain. Researchers led by Sun Ha Paek and Beom Seok Jeon at Seoul National University Hospital have identified a potential role for subthalamic deep brain stimulation (STN DBS) for the relief of pain in PD.

Paek and colleagues used a questionnaire to assess the severity and location of pain in 29 patients with PD. Each patient was assessed before and 3 months after undergoing surgical implantation of the DBS device, and 16 patients were followed-up for 6 months after surgery. Of the 29 patients, 23 reported pain before surgery. 20 of these patients reported improvements in their pain levels at 3 months postsurgery, and six reported a complete cessation. At

\begin{abstract}
6 months, 11 of the 16 patients who showed improvements at 3 months continued this trend, and two additional patients reported

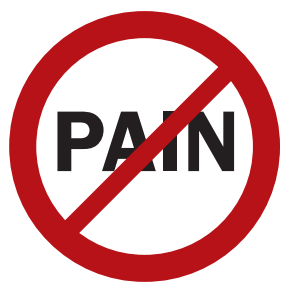
improvements for the first time.

Although these results bode well for the prospect of using STN DBS as a potential treatment for pain in PD, "Further studies are needed to evaluate the change of pain in PD with long term follow-up and it will be important to investigate the long term effect of STN DBS on pain in PD," says Paek.
\end{abstract}

\section{Mick Aulakh}

Original article Kim, H. J. et al. Chronic subthalamic deep brain stimulation improves pain in Parkinson disease. J. Neurol. 255, 1889-1894 (2009). 